\title{
Pseudoaneurisma de aorta ascendente: complicación posquirúrgica
}

\author{
Pseudoaneurysm of ascending aorta: postoperative complication
}

\author{
Leydimar Anmad-Shihadeh ${ }^{1 *}$, Marcos Farrais-Villalba', José L. Martos-Maine ${ }^{1}$, María Facenda-Lorenzo ${ }^{1}$, \\ Patricia Couto-Comba', Luís Álvarez-Acosta1 ${ }^{1}$, Rafael Llorens-León ${ }^{2}$ y Julio Hernández-Afonso ${ }^{1}$ \\ ${ }^{1}$ Servicio de Cardiología, Hospital Universitario Nuestra Señora de la Candelaria; ${ }^{2}$ Servicio de Cirugía Cardíaca, Hospiten Rambla. Santa Cruz de \\ Tenerife, Canarias, España
}

La cirugía de la aorta ascendente, mediante el uso de tubos valvulados (con la técnica de Bentall Bono desde la década de los setenta), ha sido una alternativa para los pacientes con trastornos como la anuloectasia y la disección aórtica tipo $A$, asociados o no a la enfermedad de Marfan. Sin embargo, no deja de ser una cirugía con altos riesgos de mortalidad, fundamentalmente atribuida a las complicaciones tardías, tanto inherentes a la técnica como a las secundarias (infecciones o por causas de enfermedades del tejido conectivo de la aorta primitiva), especialmente en los puntos donde conecta con las prótesis ${ }^{1}$.

Presentamos el caso de un paciente de 57 años que ingresa en nuestro hospital desde la Unidad de Imagen de la Sección de Cardiología por hallazgo ecocardiográfico. En sus antecedentes personales destacaban dos cirugías de aorta ascendente (AA), en 2008 ( $\sin$ complicaciones quirúrgicas, con técnica de Bentall Bono) y posteriormente en 2017 por insuficiencia aórtica severa con técnica de Bentall Bono, con un posquirúrgico complicado con infección, dehiscencia y hematoma de la herida quirúrgica; al sexto día del procedimiento presentó cuadro febril con hemocultivos positivos para Staphylococcus epidermidis tratándose según antibiograma y con buena evolución inicial. Sin embargo, el dieciochoavo día posquirúrgico presentó nuevamente dehiscencia de la herida quirúrgica y esternón, precisando sistema VAC, con hemocultivos de control negativos. Se realizó asimismo limpieza quirúrgica y resutura esternal con cierre de herida. Se pautó vancomicina endovenosa durante seis semanas y rifampicina $600 \mathrm{mg}$ al día, sin complicaciones. A pesar de esto, al mes volvió a reingresar por hematoma espontáneo de la herida quirúrgica, requiriendo drenaje y antibioterapia empírica con quinolonas.

En el ingreso actual, en el examen físico se objetivaron crepitantes bilaterales, ingurgitación yugular y soplo pandiastólico. Se realizó ecocardiograma transesofágico que mostró ventrículo izquierdo y ventrículo derecho en límites altos de la normalidad, ambos con contractilidad conservada; visualizándose tubo protésico en AA y prótesis mecánica aórtica con gradientes normales pero con regurgitación periprotésica severa (tiempo de hemipresión $70 \mathrm{~ms}$, vena contracta $12 \mathrm{~mm}$ ) (Fig. 1 A y B). En la región posterior a nivel supravalvular se ve imagen de dilatación entre la pared del tubo valvulado y de la aorta nativa, con expansión sistólica (flechas,

\section{Correspondencia:}

*Leydimar Anmad-Shihadeh

Servicio de Cardiología

Hospital Universitario Nuestra Señora de Candelaria

Carretera del Rosario, n. ${ }^{\circ} 145$

Fecha de recepción: 21-01-2018

Fecha de aceptación: 10-11-2018

E-mail: shihadehleydimar@gmail.com

DOI: 10.24875/ACM.M19000030
Disponible en internet: 08-05-2019 Arch Cardiol Mex. 2019;89(2):185-187 www.archivoscardiologia.com

1405-9940 @ 2018 Instituto Nacional de Cardiología Ignacio Chávez. Publicado por Permanyer México SA de CV. Este es un artículo Open Access bajo la licencia CC BY-NC-ND (http://creativecommons.org/licenses/by-nc-nd/4.0/). 


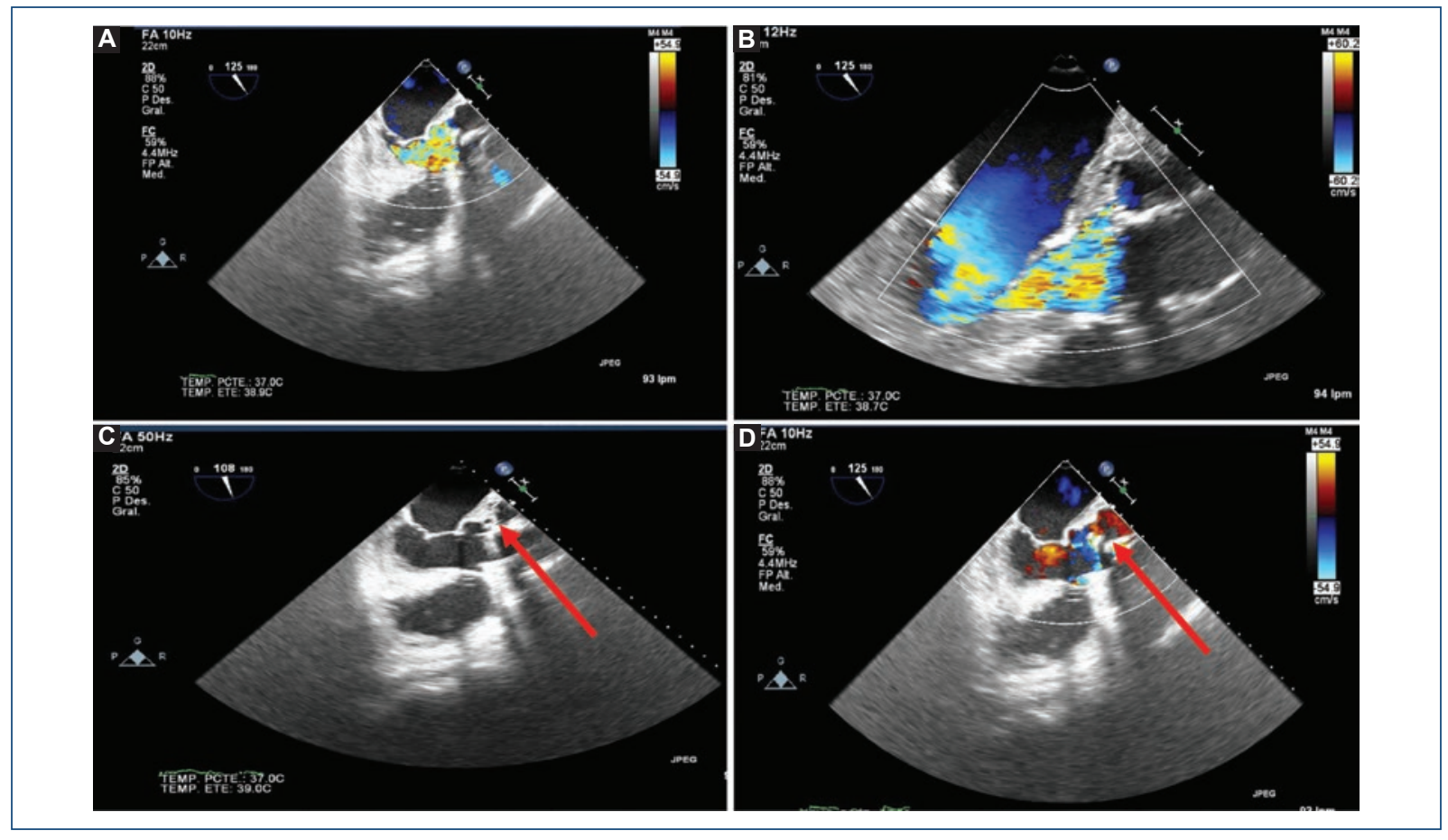

Figura 1. Regurgitación periprotésica severa (tiempo de hemipresión $70 \mathrm{~ms}$, vena contracta $12 \mathrm{~mm}$ ) (A y B). En la región posterior a nivel supravalvular se ve imagen de dilatación entre la pared del tubo valvulado y de la aorta nativa, con expansión sistólica (flechas) (C y D).

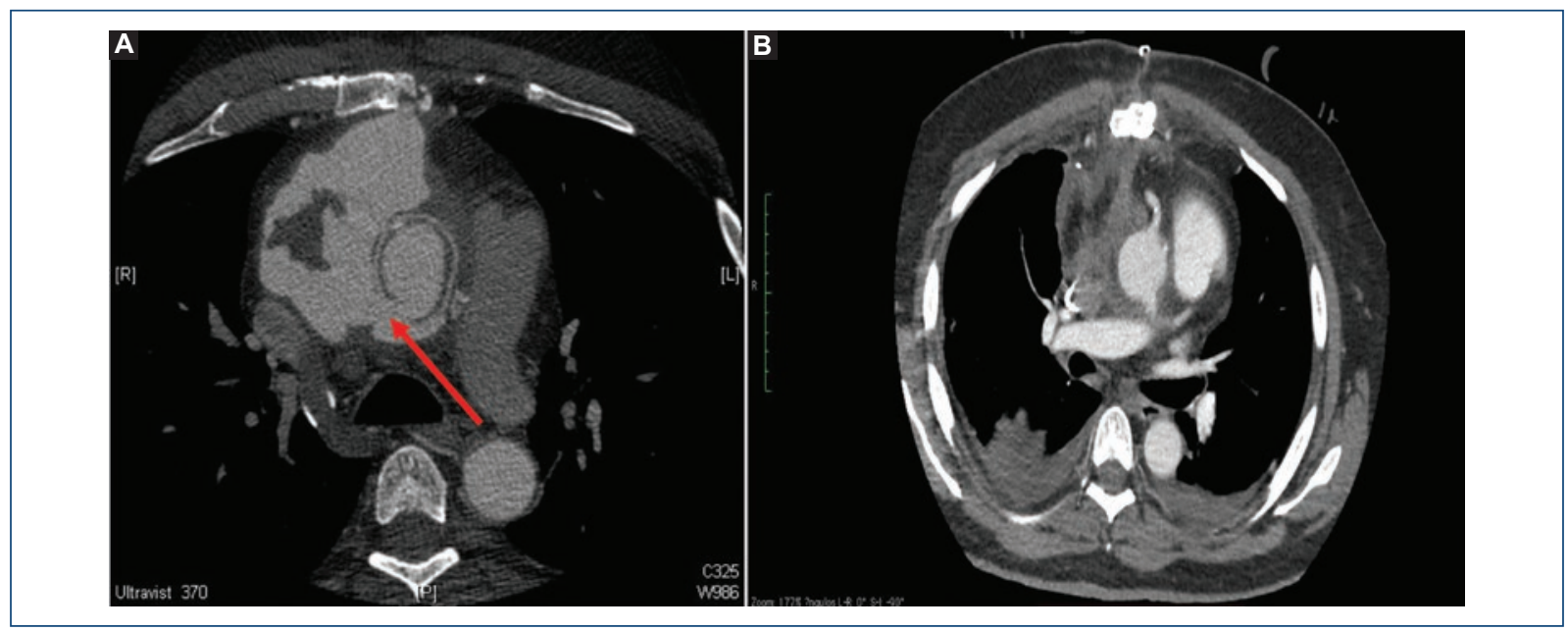

Figura 2. Tomografía computarizada: gran colección adyacente a raíz de aorta de 92 x 73 x 100 mm, con pérdida de planos con tubo protésico en su cara lateral derecha y comunicación amplia con luz aórtica, compatible con gran hematoma mediastínico secundario a rotura protésica. A: pseudoaneurisma posquirúrgico secundario a rotura protésica (flechas). B: control postoperatorio.

Fig. 1 C y D), por lo que se realizó tomografía computarizada (TC) de aorta que reveló gran colección adyacente a raíz de aorta de $92 \times 73 \times 100 \mathrm{~mm}$, con pérdida de planos con tubo protésico en su cara lateral derecha y comunicación amplia con luz aórtica, compatible con gran hematoma mediastínico secundario a rotura protésica. Estableciendo el diagnóstico por imagen de pseudoaneurisma posquirúrgico secundario a rotura 
protésica (flechas, Fig. 2A) es sometido a una intervención quirúrgica por técnica de Bentall Bono y es dado de alta sin complicaciones hasta el momento (ver TC control postoperatorio, Fig. 2B) $)^{2,3}$.

\section{Financiación}

Ninguna.

\section{Conflicto de intereses}

Ninguno.

\section{Responsabilidades éticas}

Protección de personas y animales. Los autores declaran que para esta investigación no se han realizado experimentos en seres humanos ni en animales.

Confidencialidad de los datos. Los autores declaran que han seguido los protocolos de su centro de trabajo sobre la publicación de datos de pacientes.

Derecho a la privacidad y consentimiento informado. Los autores declaran que en este artículo no aparecen datos de pacientes.

\section{Bibliografía}

1. Valenzuela García LF, Gallego García de Vinuesa P, Guerrero de Mier M, Beltrán J, Araji O, Barquero Aroca JM. Pseudoaneurisma como complicación de la reconstrucción de aorta ascendente mediante injerto valvulado: dehiscencia perianular simulando una insuficiencia aórtica severa. Rev Esp Cardiol. 1999;52:737-40.

2. Gutiérrez Sánchez JA. Cirugía de la aorta ascendente suprasinusal. Cir Cardiov. 2007;14(4):311-9.

3. Lindefjeld D, Martínez A, Heusser F, Zalaquett R. Cierre percutáneo de pseudoaneurisma post-operatorio de aorta ascendente con dispositivo Amplatzer. Rev Chil Cardiol. 2012;31:63-6. 